patients who
exhibited
intrathecal IgM
production
had an
approximately
threefold
higher risk
of converting
to MS than
those without
intrathecal IgM
production

did not exhibit intrathecal IgM production, suggesting that the disease was more aggressive in these patients.

Patients who exhibited intrathecal IgM production had an approximately threefold higher risk of converting to MS than those without intrathecal IgM production over a median follow-up period of 2 years. The risk of MS conversion associated with intrathecal IgM production tended to be higher than that associated with established radiological and CSF risk factors.

Ruprecht suggests that identifying intrathecal IgM production in patients with CIS could help guide treatment decisions. "Future research should clarify whether patients with CIS and intrathecal production of IgM derive a particularly strong benefit from starting immunotherapy early."

Sarah Lemprière

ORIGINAL ARTICLE Pfuhl, C. et al. Intrathecal IgM production is a strong risk factor for early conversion to multiple sclerosis. Neurology https:// doi.org/10.1212/WNL.0000000000008237 (2019)

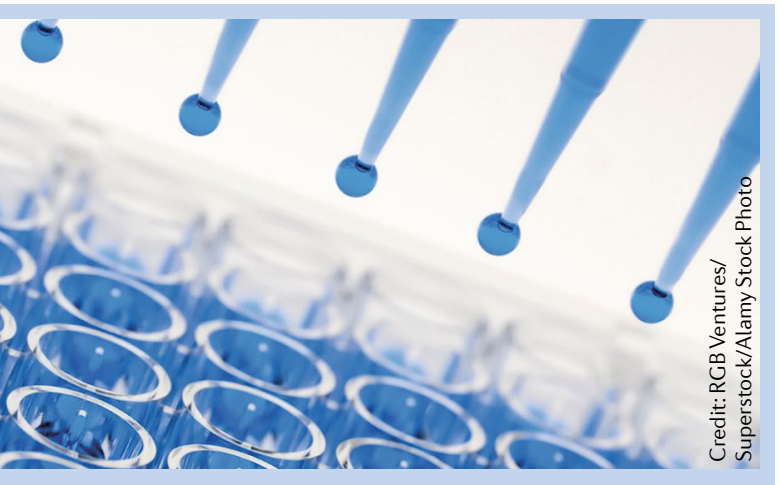

than those from saline-treated mice, and the compound had no apparent toxic effects.

"These data indicate that DHODH

4

tumours from

10580-treated

mice were

significantly

smaller than

those from saline-treated mice inhibitors are effective against $\mathrm{GIC}$ tumorigenesis and suggest 10580 as a potential new drug to treat glioblastoma," write the researchers. "The next challenge is to develop a 10580 delivery system or to find novel DHODH inhibitors that can cross the BBB."

Sarah Lemprière

ORIGINAL ARTICLE Echizenya, S. et al. Discovery of a new pyrimidine synthesis inhibitor eradicating glioblastoma-initiating cells. Neuro-Oncology https://academic.oup.com/neurooncology/ advance-article/doi/10.1093/neuonc/noz170/ 5566464 (2019)

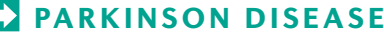

\section{Could a prostate drug be repurposed for Parkinson disease?}

Terazosin, a drug that enhances glycolysis and is used to treat benign prostatic hyperplasia (BPH), has the potential to be repurposed for the treatment of Parkinson disease (PD), according to a new study published in The Journal of Clinical Investigation. An international team of researchers gathered evidence from multiple sources, including animal and cellular models of PD and human databases, to build up a profile of terazosin as a possible disease-modifying drug for PD.

"Current medicines can partially alleviate some of the symptoms of PD, but today we have zero treatments that change the progressive course of this neurodegenerative disease," comments Michael Welsh from the University of lowa, USA, who was one of the leaders of the study. "That's a terrible state, because as our population ages, $\mathrm{PD}$ is going to become increasingly common."

Terazosin works by increasing the activity of phosphoglycerate kinase 1 (PGK1) - a key ATP-generating enzyme in the glycolytic pathway. The decision to investigate terazosin in the context

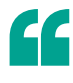

When we tested the drug in various different animal models of PD, they all got better

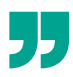
of PD stemmed from observations of energy impairment in patients with this condition.

The researchers tested terazosin in toxin-induced and genetic forms of PD in rodents and flies. The drug was shown to increase energy production, reduce neurodegeneration and partially restore brain dopamine levels and motor function in these models. Beneficial effects of terazosin on neuronal survival and PD pathology were also demonstrated in induced pluripotent stem cell-derived dopamine neurons from patients with PD.

"When we tested the drug in various different animal models of PD, they all got better," reports co-corresponding author Lei Liu, who is based at Capital Medical University, Beijing, China. "Both the molecular changes in the brain associated with cell death and the motor coordination in the animals improved."

To further investigate the effects of terazosin on PD in humans, Nandakumar Narayanan and Jordan Schultz from the University of lowa turned their attention to clinical databases. In patients with PD from the Parkinson's Progression Markers Initiative database, the use of terazosin (or similar drugs that enhanced PGK1 activity) was associated with a reduced rate of motor decline. In addition, findings from the IBM Watson/Truven database suggested that these drugs decreased the risk of PD in the wider population.

The researchers used tamsulosin, a drug that is prescribed for BPH but that does not affect PGK1 activity, as a comparator in their database analyses. Tamsulosin did not seem to affect the risk or disease course of PD, implying that the beneficial effects of terazosin were being mediated through PGK1.

"What is particularly exciting is that terazosin is a 'repurposed drug', so we already have a lot of safety data from its clinical use to treat enlarged prostate," explains Narayanan. "We are currently engaged in planning phase I studies - the beginning of what we hope will be a sustained and rigorous effort to test this molecule prospectively."

Heather Wood

ORIGINAL ARTICLE Cai, R. et al. Enhancing glycolysis attenuates Parkinson's disease progression in models and clinical databases. J. Clin. Invest. https://doi.org/10.1172/JCl129987 (2019)

RELATED ARTICLE Elkouzi, A. et al. Emerging therapies in Parkinson disease - repurposed drugs and new approaches. Nat. Rev. Neurol. 15, 204-223 (2019) 